\title{
Data archiving in animal experimentation: Merits, challenges, and a case study
}

\author{
JONATHON D. CRYSTAL \\ University of Georgia, Athens, Georgia
}

\begin{abstract}
Electronic data archives may supplement the traditional peer-reviewed journal article. The merits of data archiving include public service, a more complete research project, overcoming barriers to limitedaccess research resources, and increasing the impact of a scientific project. A case study of chimpanzee timing performance in space is derived from the NASA Life Sciences Data Archive (http://lsda.jsc. nasa.gov). An analysis of the archived data suggests that the scalar property (a form of Weber's law) applies to timed performance of a chimpanzee in orbit of the earth. Challenges associated with data archives are discussed. Although significant challenges are associated with archiving electronic data, these difficulties are outweighed by its merits.
\end{abstract}

The development of a scientific project begins with the inception of a research question and typically ends with the publication of a journal article reporting the outcome of a study. The ongoing impact of the project is measured by citations of the journal article and, perhaps, the reproduction of some of the data (e.g., a figure from the original article might appear in a subsequent review article). The impact of a research project may be substantially increased by archiving the original data (and associated materials, such as stimuli). Developments in Web-based technology make it feasible to archive large amounts of materials associated with a research project. For example, it is possible to make available all of the data in their most raw form (e.g., an electronic list of the time of occurrence of all stimuli and responses for each subject during each test session for each experiment). The leadership of the Psychonomic Society in facilitating archiving of data associated with its publications may establish electronic archives as a standard adjunct to the traditional peerreviewed journal article. In addition, developments in subdisciplines of psychology (e.g., animal cognition; Church, 2002; Kurtzman, Church, \& Crystal, 2002) may foster the widespread establishment of data archives.

This article will evaluate the merits and challenges of data archiving in the subdiscipline of animal-cognition research. Archiving in animal research does not require a resolution of the privacy and confidentiality issues associated with research on human subjects. Chimpanzee timing performance in space will be considered as a case study, using a data archive established by the National Aeronautics and Space Administration (NASA).

Preparation of this article was supported by National Institute of Mental Health Grant MH64799. I thank Russ Church for his comments on the manuscript. The raw data, provided by NASA, are available at http:// 1sda.jsc.nasa.gov and at www.uga.edu/animal-cognition-lab. Correspondence concerning this article should be addressed to J. D. Crystal, Department of Psychology, University of Georgia, Athens, GA 30602-3013 (e-mail: jcrystal@uga.edu).

\section{Merits of Data Archiving}

There are several merits to archiving data associated with a peer-reviewed journal article. Electronic data archives provide an efficient method to share data, encouraging scientific inquiry and the diversity of methods of data analysis. Access to raw data will promote testing new hypotheses and analytic methods, permitting the exploration of hypotheses not envisioned by the initial investigators. Other merits of data archiving include serving the public, a more complete product than what appears in a traditional journal article, overcoming limited access to research resources, and increasing the impact of a scientific project.

Public service. To a large extent, research in psychology is funded by the government. The mission in this work is to develop knowledge and make it publicly available. This knowledge has traditionally come in the form of distilled selections of the research effort (Latour, 1987). For example, the data that typically appear in a journal article have been averaged first within subjects and then across subjects. Of course, it is not possible to recover the raw data from the journal article, but it would be valuable for others (e.g., researchers, students, the public) to be able to obtain more than the selection offered by the investigators.

The impetus to make data available to the public via electronic archives comes from values, policies, and politics. There is a scientific value for open inquiry. For example, scientific societies (e.g., the American Psychological Association [APA], 2002) establish ethical principles and scientific practices that require authors to make raw data and associated materials available upon request. The APA ethical standards of sharing research data for verification (Standard 8.14) states:

\footnotetext{
After research results are published, psychologists do not withhold the data on which their conclusions are based from other competent professionals who seek to verify the substantive claims through reanalysis and who intend to use such data only for that purpose ....
} 
This standard is implemented in APA journals by requiring authors to keep their data available for at least 5 years after the date of publication. Making data archives publicly available would satisfy and exceed these requirements.

Because of the widespread use of government funds, the data belong to the public, rather than to the investigator who collected them. The investigator has the initial right to access the data before making them publicly available, but this is not a permanent right to restricted access. An electronic data archive represents an efficient long-term venue for making the products of research widely available.

Policies of funding agencies increasingly emphasize the sharing of data. For example, the National Institutes of Health (NIH) has established a policy (http://grants2.nih. gov/grants/policy/data_sharing/data_sharing_guidance. $\mathrm{htm}$ ) according to which all data should be considered for data sharing, and all applications for relatively large grants are required to provide a plan to share final research data; a data-sharing plan is required for grant applications seeking $\$ 500,000$ or more in direct costs in any one year of the application. Similarly, the National Science Foundation (NSF) has established a policy according to which investigators are expected to share data and other materials with other researchers (NSF 03-2 Grant Proposal Guide Chapter VI, Section H; http://www. nsf.gov/pubs/2003/nsf032/032_6.htm\#VIH).

The policies of funding agencies are being established in response to political movements to make data available to the public. For example, implementation of the Freedom of Information Act (FOIA) has recently been modified to permit public requests for research data. Currently, these provisions apply only to data produced with support from the federal government if the data are cited by a federal agency in establishing a regulation that has the effect of law. However, it is conceivable that the FOIA might be revised further to permit requests for other data. In addition, although significant pieces of research are published in psychological journals without the use of public funds, government agencies may impose the requirement to share data on all projects that are produced by an institution that accepts public funds. This type of mandate might require an institution to adhere to federal standards in all of its research whenever it accepts federal funds for a subset of its research; for example, this is common in setting standards for research on human and nonhuman subjects. Many states have open-record laws for government agencies, which could be used by the public to request documents associated with completed research projects at public universities. The outcome is a political trend to require that data be made available to the public.

In summary, there are both ethical and legal rationales for archiving data and making them publicly available. The moral argument is that making data available is a service to the public. The legal argument is that the government and funding agencies are increasingly requiring dissemination of data.
A complete research product. Depositing raw data in an electronic archive associated with a journal article represents a more complete research report than a journal article alone. The journal article is complemented by the archiving of raw data, stimuli used in the experiments, data analysis programs, programs used to conduct simulations and test theories, pictures or video of equipment and testing environments, and/or other materials. In general, the ability to include such materials in the traditional journal article is limited by the number and cost of pages and the print format of traditional journals. A complete list of raw data would be hard to include in a traditional journal article. Moreover, a large amount of data in printed form is less accessible than the same data in electronic form.

Electronic archiving of supplementary materials may lead to new insight into research questions. For example, the ability to examine the original stimuli that were used in a published experiment may lead to new alternative hypotheses about the phenomena in the article. New experiments may be conducted with the archived and/or new stimuli that would otherwise not be possible. In addition, the availability of the original stimuli permits more exact replications of critical experiments. Archiving data from animal research may be considered a component of a complete research product without resolving the issues of privacy and confidentiality associated with research on human subjects.

Limited access to research resources. Conducting psychological research often requires access to considerable resources. Resources required for animal-cognition research include live animals, animal-housing facilities, food, animal-care staff, veterinarians, equipment for behavioral testing, computers, and software. In addition, conducting animal research typically requires substantial investments in time and funds. It is generally not possible to conduct animal research without all of these resources. Consequently, the number of individuals pursuing research questions is limited by the availability of resources. If investigators who have access to these limited resources deposit data into electronic archives, other individuals may pursue research questions by analyzing the available data. This is particularly helpful for training students. For example, in an undergraduate or graduate course, the ability to generate new data is constrained by time and available resources. The range of experiences fostered in student training may be increased by analyzing authentic data sets from archives. Data archives may also enhance the experience of undergraduates engaged in independent research projects; students can be encouraged to analyze archived data before designing and conducting new experiments. Consequently, access to raw data could enhance instruction and the training of new investigators.

The use of animals in research provides an additional incentive to archive data. Ethical principles (e.g., Guide for the Care and Use of Laboratory Animals, 1996; Guidelines for Ethical Conduct in the Care and Use of Animals, 
1996) require that the number of animals used in research be minimized. Access to data from previous experiments may reduce the number of animals used in ongoing research projects. For example, the number of redundant control conditions may be reduced by reanalyzing archived data. Alternatively, it may be necessary to conduct some control conditions simultaneously with new experimental conditions. However, the sample sizes in these conditions may be reduced by combining data from new and archived sources. Consequently, the availability of archived data may represent a new way to reduce the number of animals required to address a research question.

Impact of a scientific project. If a scientific project ends with the publication of a journal article, the impact of the project is limited to what the investigator chose to include in the article. In contrast, if the investigator deposits the raw data into an electronic archive, other investigators can ask new questions that were not envisioned by the initial investigators. This is particularly important as new theories develop and emerge over time. A new theory or prediction may be appropriately tested from a previously published experiment if the raw data are available for examination. Testing these new predictions can increase the impact of a scientific project in ways that might not have been anticipated when the journal article was published. For example, a research question may be pursued by combining multiple data sets from independent data archives. This may be accomplished by meta-analysis (e.g., Mullen, 1989), which involves combining statistical information from multiple studies, or from the secondary analysis of archived data; a weakness of meta-analysis is that it is limited to the statistical analysis of published data (Church, 2002). An archived data set could have an impact on new, as yet unidentified, developments that are not possible for the traditional journal article. For example, performance in an experiment is often summarized in a journal article by presenting means across subjects. Information about the variability within subjects is lost in this presentation. After a traditional journal article is published, a new theory's prediction about variability cannot be tested unless the experiment is repeated. If the raw data are available in an electronic archive, predictions about variability or the shape of response distributions for each subject can be tested. This issue will be illustrated with a case study.

\section{Case Study: Chimpanzee Timed \\ Performance in Space}

On November 29, 1961, NASA launched the flight of Mercury-Atlas 5 (MA-5) as a prelude to the first manned orbital space flight. Enos, a 5.25-year-old male chimpanzee (Pan troglodytes), was on board for the $3.35-\mathrm{h}$ flight that orbited the earth twice before returning. Enos had received 16 months of operant training prior to the flight. The objectives of the mission included the collection of data on the psychological demands that would be encountered by astronauts during orbital space flights. The NASA Life Sciences Data Archive (http://lsda.jsc. nasa.gov) includes the raw data from the operant exper- iments (in addition to details about the flight and Enos's physiological state). Publications associated with the MA-5 mission include Henry and Mosley (1963), Rohles, Grunzke, and Reynolds (1962), and Wheelwright (1962).

The purpose of the operant experiments was to determine whether the restrained chimpanzee could respond normally in the weightless state, to assess motivation for food and water, and establish that eating and drinking could be carried out during weightlessness. The operant experiments included continuous avoidance and discrete avoidance, a differential reinforcement of low rate (DRL) schedule, a fixed-ratio schedule of reinforcement, and an oddity visual discrimination. The conclusions that emerged from the mission were that visual and tactile performance were unimpaired and that the psychological demands that astronauts would encounter during space flight would not be excessive.

Although the purpose of the operant testing was to assess the psychological demands of orbital space flight prior to sending John Glenn on the first manned orbital space flight, the raw data are available to test predictions of psychological theories, including theories that did not exist when the data were collected. To illustrate this point, I examined the raw data (interresponse intervals) from the DRL schedule to determine whether the scalar property (a form of Weber's law; see, e.g., Gibbon, 1977) is exhibited in space. The scalar property is a feature of timed performance in which the coefficient of variation (standard deviation divided by the mean of a distribution) of timed performance is constant as a function of interval conditions. The scalar property has received extensive empirical support (e.g., Gibbon, 1991), although counterexamples have been documented (e.g., Crystal, 1999, 2001a, 2001b, 2003, 2004); the counterexamples come from testing many closely spaced interval conditions, which reveals local maxima in sensitivity to time. The scalar property of timing was first documented in 1970 (Catania, 1970; Dews, 1970; Gibbon, 1991), and its theoretical significance was first emphasized in 1977 (Gibbon, 1977), more than a decade after the data were collected on MA-5. The scalar property is predicted by various contemporary timing theories (e.g., Church \& Broadbent, 1990; Church, Meck, \& Gibbon, 1994; Gallistel \& Gibbon, 2000; Gibbon, 1977; Killeen \& Fetterman, 1988).

In order to test this prediction with the NASA data, the mean, standard deviation $(S D)$, and coefficient of variation $(\mathrm{CV})$ of interresponse intervals were calculated for each of the three sessions that were conducted on MA-5; water was delivered as a reward contingent on an interresponse time that was $20 \mathrm{sec}$ or longer whenever a green light was illuminated. Figure 1 shows mean, $S D$, and $C V$ plotted as a function of sessions. Note that the mean and $S D$ declined on the third session, but the $\mathrm{CV}$ remained relatively constant across sessions. The constant $\mathrm{CV}$ is consistent with the scalar property. Although the scalar property is typically evaluated as a function of widely spaced interval conditions, in this case it is evaluated as a function of performance changes within a single experimental condition. 

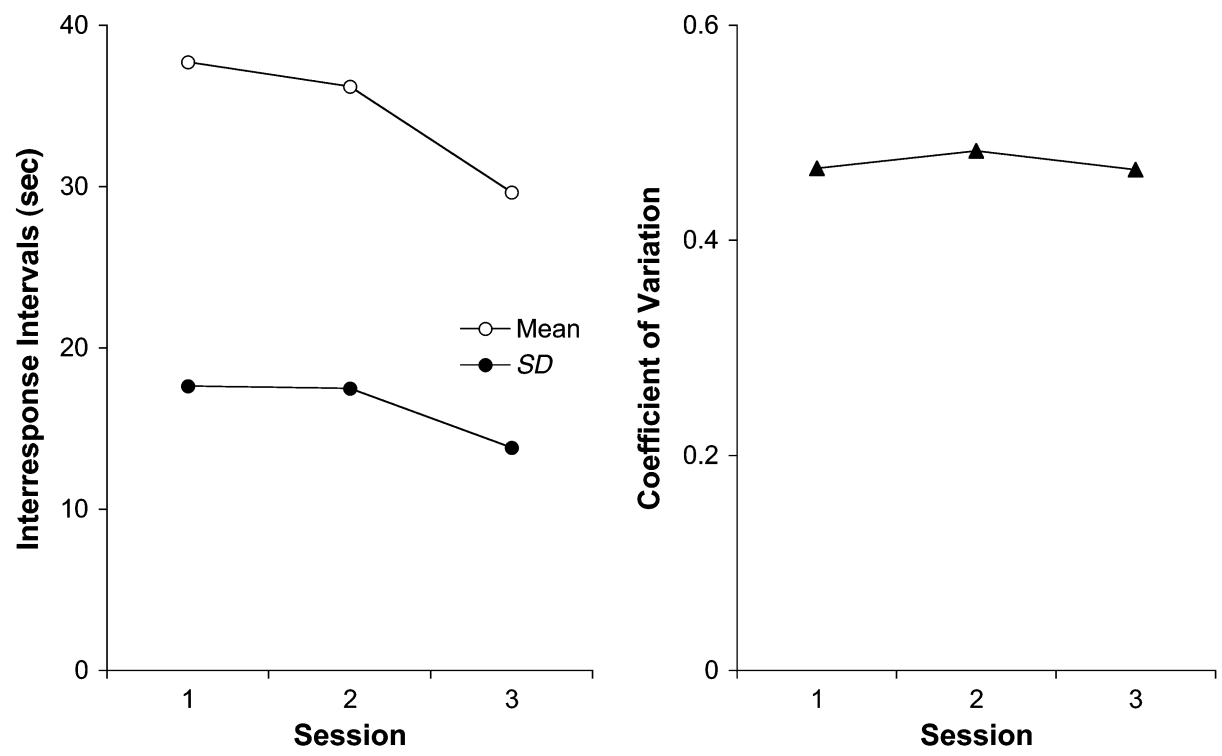

Figure 1. Mean and standard deviation $(S D)$ of interresponse intervals are plotted as a function of sessions (left panel), and coefficient of variation $(\mathrm{CV}, S \mathrm{SD} / \mathrm{mean})$ is plotted as a function of sessions (right panel). The data were obtained from the NASA Life Sciences Data Archive (http://lsda.jsc. nasa.gov) and describe the performance of a chimpanzee pressing a lever under a differential reinforcement of low rate schedule.

This case study illustrates the merit of electronic data archives. This is an extreme case of limited access to research resources. It is rare to launch a chimpanzee into orbit around the earth. The case study also illustrates the merit of archiving data in its most raw form. Although an examination of the variability of timed performance was not a focus of the research project at the outset, the availability of raw data permits subsequent analyses to be performed. The subsequent examination of $\mathrm{CV}$ as a test of the scalar property was not anticipated at the time of data collection. The NASA Life Sciences Data Archive includes the raw data, pictures of the apparatus for testing the chimpanzee, details about the testing environment (e.g., G-force acceleration) that can be correlated with the time at which behavioral testing was conducted, and physiological measures of the chimpanzee throughout the mission.

The case study also illustrates some of the challenges associated with data archiving. For example, it would be desirable to evaluate $\mathrm{CV}$ as a function of different DRL schedules (e.g., DRL 20 vs. $40 \mathrm{sec}$ ). It would also be desirable to have data from more than one subject. Although the NASA Life Sciences Data Archive provides raw data for the performance of the chimpanzee during the MA-5 mission, no data are available to describe the preflight (or postflight) performance in the operant experiments. The secondary data analysis is limited by the original experimental design.

\section{Challenges Associated With Data Archiving}

Despite the merits of electronic data archiving, it presents several potential challenges. First, details about the original experiment may be incomplete. This problem may be exacerbated when the secondary data analysis pursues a question that was not anticipated at the time the data were collected and archived; in this case, some details of the experimental design may not have been considered central enough to describe. In the case study, for example, insufficient details about training or testing before or after the MA-5 mission are provided in the NASA archive.

Second, the original experimental design may not be adequate to answer new research questions. For example, experimental manipulations in the original design may not have been sufficient. In the case study, the ability to evaluate the scalar property is improved by experimentally manipulating the DRL schedule's parameter, but data are not available from different DRL schedules.

Third, there is a potential problem of overrelying on a small number of data sets. Any problems associated with these data sets would be magnified by relying on subsequent analyses of these data. In an extreme case, one might propose to conduct a single experiment on a research problem; subsequent analysis would be conducted with this single data set. In another extreme case, one might propose to conduct a new experiment each time a new hypothesis was proposed for a given research problem. It is likely that a balance between these two extreme cases can be achieved so that one does not rely too much on a single data set or needlessly repeat an experiment many times over.

One of the merits of electronically archiving data is public service. However, a fourth challenge is associated with achieving this goal. In particular, it is possible that segments of the public might misinterpret the materials in an electronic archive. For example, a video archive of animal research might be used by an animal rights ac- 
tivist in a selective or misleading way that could not have been anticipated at the time that the materials were prepared for archiving.

A fifth challenge associated with electronic archives is to encourage researchers to archive the data associated with the journal articles that they publish. Relatively few laboratories in animal cognition are currently archiving data or other materials.

Long-term preservation of archived materials represents a sixth challenge for efforts to archive data. It is desirable to have archived data available in perpetuity. Resources are required to achieve this goal (e.g., time, computer resources, updating to new technologies for access to data, links to other resources within a database, etc.). Perhaps NASA can guarantee preservation, but how can individual investigators achieve this type of preservation? Universities, libraries, and scientific societies will need to play a critical role in ensuring preservation of electronic archives. The Psychonomic Society has established an electronic archival repository for materials associated with its publications.

A final challenge involves finding existing electronic archives. Because electronic archives exist in a distributed network (individual labs, universities, libraries, scientific societies, other consortia, etc.), finding existing archives represents a challenge. Centralized archives could reduce this problem. Alternatively, a centralized list of distributed data archives would be helpful.

The case study illustrates some of the difficulties associated with finding existing electronic archives. I found the NASA Life Sciences Data Archive by using a Webbased search engine. However, this method has several limitations. First, a search for data archives in animal cognition yields too many hits, many of which are not relevant. Moreover, it is possible that valuable archives will fail to be identified in such a search. Alternatives to Web-based searches include supplements to journal articles and organizational databases in specific disciplines or subdisciplines.

Each of the challenges described above can be overcome. Some of the challenges have scientific solutions (1-3 above); some have political solutions (4-5); and some have organizational solutions (6-7).

\section{Conclusions}

Electronic data archives have the potential to increase access to the research product and to increase the impact of a research project. The data from an archived project can be used to answer unexpected questions, thereby extending the value of the project after the publication of a traditional peer-reviewed article. Data archives represent an important service to the research community and, more broadly, to the public. Data archiving in animal cognition is especially valuable given the limited access to animal-research resources and the requirement to minimize the number of animals used in research. Although significant challenges are associated with archiving electronic data, these difficulties are outweighed by its merits.

\section{REFERENCES}

American Psychological Association (2002). Ethical principles of psychologists and code of conduct. American Psychologist, 57, 10601073 .

Catania, A. C. (1970). Reinforcement schedules and psychophysical judgments: A study of some temporal properties of behavior. In W. N. Schoenfeld (Ed.), The theory of reinforcement schedules (pp. 1-42). New York: Appleton-Century-Crofts.

Church, R. M. (2002). The effective use of secondary data. Learning \& Motivation, 33, 32-45.

Church, R. M., \& Broadbent, H. A. (1990). Alternative representations of time, number, and rate. Cognition, 37, 55-81.

Church, R. M., Meck, W. H., \& Gibbon, J. (1994). Application of scalar timing theory to individual trials. Journal of Experimental Psychology: Animal Behavior Processes, 20, 135-155.

CRYSTAL, J. D. (1999). Systematic nonlinearities in the perception of temporal intervals. Journal of Experimental Psychology: Animal Behavior Processes, 25, 3-17.

Crystal, J. D. (2001a). Circadian time perception. Journal of Experimental Psychology: Animal Behavior Processes, 27, 68-78.

Crystal, J. D. (2001b). Nonlinear time perception. Behavioural Processes, 55, 35-49.

CRystal, J. D. (2003). Nonlinearities in sensitivity to time: Implications for oscillator-based representations of interval and circadian clocks. In W. H. Meck (Ed.), Functional and neural mechanisms of interval timing (pp. 61-75). New York: CRC Press.

CRYSTAL, J. D. (2004). Sensitivity to time: Implications for the representation of time. Manuscript submitted for publication.

DEws, P. B. (1970). The theory of fixed-interval responding. In W. N. Schoenfeld (Ed.), The theory of reinforcement schedules (pp. 43-61). New York: Appleton-Century-Crofts.

Gallistel, C. R., \& Gibbon, J. (2000). Time, rate, and conditioning. Psychological Review, 107, 289-344.

GibBon, J. (1977). Scalar expectancy theory and Weber's law in animal timing. Psychological Review, 84, 279-325.

GibBon, J. (1991). Origins of scalar timing. Learning \& Motivation, 22, 3-38.

Guide for the care and use of laboratory animals (1996). Washington, DC: National Academy Press.

Guidelines for ethical conduct in the care and use of animals [Brochure] (1996). Washington, DC: American Psychological Association.

Henry, J. P., \& Mosley, J. D. (EDs.) (1963). Results of the Project Mercury ballistic and orbital chimpanzee flights: National Aeronautics and Space Administration (NASA Special Publication SP-39). Washington, DC: Office of Scientific and Technical Information, NASA.

Killeen, P. R., \& Fetterman, J. G. (1988). A behavioral theory of timing. Psychological Review, 95, 274-295.

Kurtzman, H. S., Church, R. M., \& Crystal, J. D. (2002). Data archiving for animal cognition research: Report of an NIMH workshop. Animal Learning \& Behavior, 30, 405-412.

LATOUR, B. (1987). Science in action: How to follow scientists and engineers through society. Cambridge, MA: Harvard University Press.

Mullen, B. (1989). Advanced BASIC meta-analysis. Hillsdale, NJ: Erlbaum.

Rohles, F. H., GrunzKe, M. E., \& Reynolds, H. H. (1962). A detailed account of chimpanzee performance during the ballistic and orbital Project Mercury flights (Technical Documentary Report ARL-TDR62-15). Holloman AFB, NM: Aeromedical Research Laboratory.

Wheelwright, C. D. (1962). Physiological sensors for use in Project Mercury (NASA Technical Note TN D-1082). Houston, TX: National Aeronautics and Space Administration, Lyndon B. Johnson Space Center.

(Manuscript received November 19, 2003; revision accepted for publication July 30, 2004.) 